\title{
Determination of Zoledronic Acid and Four Common Anions in Zoledronic Acid Injection by Gradient Ion Chromatography with a Self-Regeneration Suppressor
}

\author{
Meilan Chen ${ }^{*}$, Mingli Ye ${ }^{2}$ \\ ${ }^{1}$ College of Biology and Environmental Engineering, Zhejiang Shuren University, Hangzhou, China \\ ${ }^{2}$ Shanghai Lab., Thermofisher Scientific, Shanghai, China \\ Email: ${ }^{*}$ rain-lake@163.com
}

Received 7 August 2014; revised 19 September 2014; accepted 22 October 2014

Copyright (C) 2014 by authors and OALib.

This work is licensed under the Creative Commons Attribution International License (CC BY). http://creativecommons.org/licenses/by/4.0/

(c) (i) Open Access

\begin{abstract}
A simple method to determine zoledronic acid and impurities in drug by ion chromatography with conductivity detection was developed. Target analytes have been sufficiently separated from each other on an Ionpac AS19 by using potassium hydroxide as eluent with a self-regeneration suppressor. This novel proposed method enables direct analysis of zoledronic acid in injection sample and provides good resolution. This novel method also exhibits satisfactory precision (less than $1.68 \%)$ and accuracy $(90.0 \%-110.0 \%)$, good linearity $\left(r^{2}=0.9993-0.9999\right)$ and sufficient sensitivity $(0.007-0.030 \mu \mathrm{g} / \mathrm{mL})$. It can be easily and conveniently adopted for the routine quality control analysis.
\end{abstract}

Keywords

Zoledronic Acid, Ion Chromatography, Self-Regeneration Suppressor

Subject Areas: Analytical Chemistry, Environmental Sciences

\section{Introduction}

Phosphor is of interest because it plays an important role in organism metabolism. Since 80's of the last century, some bisphosphonates (BPs), such as pamidronic acid, zoledronic acid, alendronic acid, ibandronic acid and so on, were developed and used as bone resorption inhibitors drugs and prevention of skeletal related events in patients [1]. In these BPs, zoledronic acid (1-hydroxy-(1H-imidazol-1-yl)-phosphonoethyl) which has the most pharmacological activity so far and belongs to the third bisphosphonates drugs can inhibit osteoclast activity ef-

How to cite this paper: Chen, M.L. and Ye, M.L. (2014) Determination of Zoledronic Acid and Four Common Anions in Zoledronic Acid Injection by Gradient Ion Chromatography with a Self-Regeneration Suppressor. Open Access Library Journal, 1: e827. http://dx.doi.org/10.4236/oalib.1100827 
ficiently and is used to prevent skeletal fractures in patients with cancers such as multiple myeloma and prostate cancer, as well as for treating osteoporosis [2]. So, simple, reliable and sensitive analytical methods to determine zoledronic acid are necessary for the quality control of these drugs. However, BPs have strong polarity and are very difficult to retain in the reverse phase HPLC (RP-HPLC) column. Moreover, zoledronic acid with imidazole group only has weak ultraviolet absorption. Therefore, up to now, only two techniques have been reported to determine zoledronic acid in drugs: ion-pair RP-LC with evaporative light-scattering detector [3], ion-pair RP-LC with ultraviolet detection [4]. However, evaporative light-scattering detector had to use volatile organic amine as mobile phase additive and ion-pair RP-LC with ultraviolet detection has lower sensitivity because of lack chromophore for detection.

Recently, ion chromatography (IC) has become a routine analytical method for the determination of inorganic anions and organic acids in various matrices. Although, anion-exchange IC with post-column indirect fluorescence detection was applied to the determination of bisphosphonates [5], it is complex and inconvenient.

ICS-2000 (reagent-free IC system) with eluent generation has a number of advantages because this system can minimize unintentional variations in the preparation of eluents and regeneration solution. The eluent is achieved by a $\mathrm{KOH}$ eluent generator and only deionized water needs to be supplied by the user, conductivity background is low, and gradients are more reproducible, manual error or potential problems with the proportioning valve in the eluent pump are not being factors [6]-[7].

In this work, four common anions and zoledronic acid were determined simultaneously by a reagent-free IC (ICS-2000) with a self-generation suppressor. Finally, the proposed method was successfully applied to analysis of commercial injection sample.

\section{Experimental}

\subsection{Instrumentation}

A reagent-free Dionex (Dionex, Sunnyvale, CA, USA) Model ICS-2000 ion chromatograph equipped with a 25 $\mu \mathrm{L}$ loop was used. The guard and analytical column were Dionex IonPac AG19 (50 mm $\times 4 \mathrm{~mm}$, I.D) and AS19 (250 mm $\times 4 \mathrm{~mm}$, I.D), respectively. The eluent was achieved by a Dionex Reagent-Free Controller (RFC-30) and a KOH eluent generator. The suppressor was achieved by a Dionex ASRS-300 self-regenerating membrane suppressor. The eluent flow rate was $1.0 \mathrm{~mL} / \mathrm{min}$. The injection volume was $25 \mu \mathrm{L}$. All the instruments control and data collection were performed with Chromeleon 6.0 software (Dionex, USA).

\subsection{Reagents}

All the analytical chemicals were of analytical reagent grade. Deionized water of $18.2 \mathrm{M} \Omega \cdot \mathrm{cm}$ achieved by a Millipore water system (Millipore, Mosheim, France) was used throughout. The standard stock solution of zoledronic acid was obtained from National Research Center for Certified Reference Materials (Beijing, China). The standard mixture was prepared by diluting these stock solutions and to be further diluted to the appropriate concentration $(0.1-80 \mu \mathrm{g} / \mathrm{mL})$.

\subsection{Pretreatment of Real Sample}

A $5.0 \mathrm{~mL}$ of zoledronic acid injection (purchased from local drug shop) was transfer into a $100 \mathrm{~mL}$ volumetric flask and diluted to $100 \mathrm{~mL}$ with deionized water, which was shaked thoroughly, and then centrifuged for 5 min at $5000 \mathrm{r} / \mathrm{min}$. The above solution was filtered through C18 cleanup column and a $0.45 \mu \mathrm{m}$ syringe filter before injection.

\section{Results and Discussion}

\subsection{Optimization of Separation Conditions}

Zoledronic acid and metaphosphoric acid are strongly retained and thus high concentration of $\mathrm{KOH}$ solution has to be used to elute them. On the other hand, lower concentration of $\mathrm{KOH}$ should be maintained in order to separate four anions, such as $\mathrm{Cl}^{-}, \mathrm{SO}_{4}^{2-}, \mathrm{PO}_{4}^{3-}$, and so on. Therefore, gradient eluent program and high hydrophilic and capability column (IonPac AS19) were used. The gradient program of eluent was listed in Table 1. Under the selected experimental conditions, the five anions including chloride, carbonate, sulfate, phosphate and hexametaphosphate did not interfere with the determination of zoledronic acid. Figure 1 is a chromatogram of a 
Table 1. The gradient program of $\mathrm{KOH}$ eluent.

\begin{tabular}{cc}
\hline Time (min) & Concentration of $\mathrm{KOH}$ solution $(\mathrm{mmol} / \mathrm{L})$ \\
\hline 0.0 & 30 \\
7.0 & 30 \\
10.0 & 70 \\
13.0 & 70 \\
13.1 & 30 \\
\hline
\end{tabular}

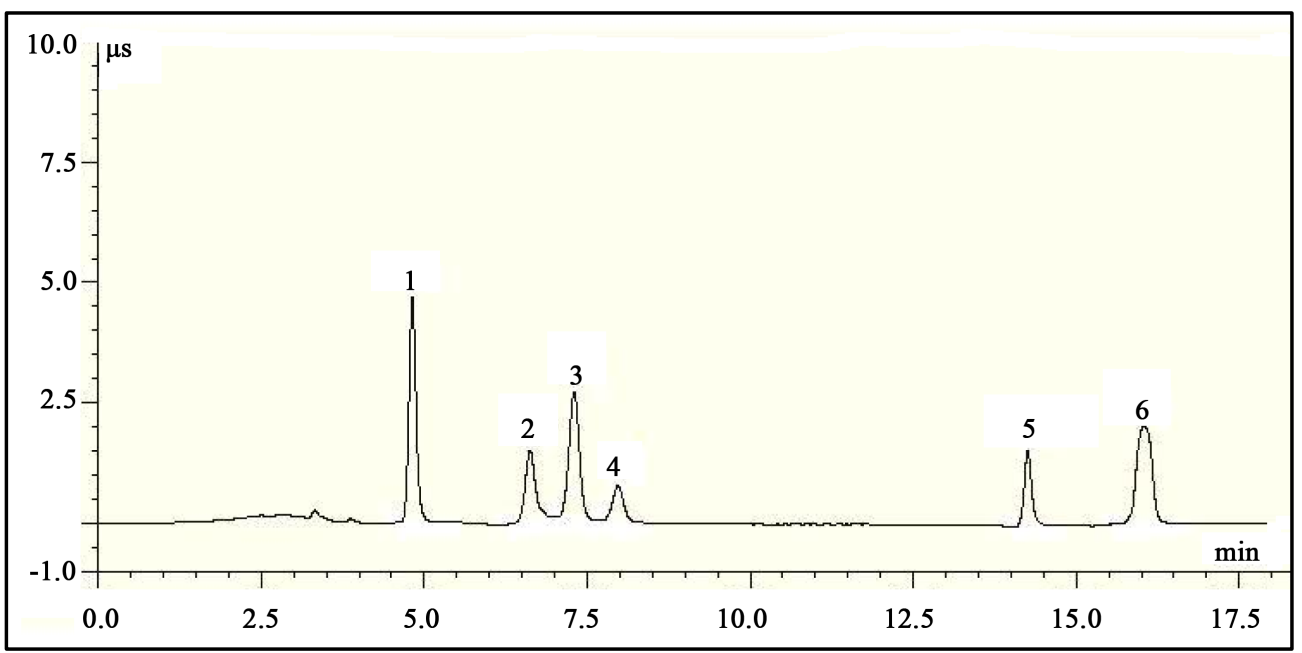

Figure 1. Chromatogram of the standard of zoledronic acid and five anions. Peaks identified as: 1 chloride $(2 \mu \mathrm{g} / \mathrm{mL}) ; 2$ - carbonate $(2 \mu \mathrm{g} / \mathrm{mL}) ; 3$-sulfate $(2 \mu \mathrm{g} / \mathrm{mL}) ; 4$-phosphate $(3 \mu \mathrm{g} / \mathrm{mL}): 5$ hexametaphosphate $(20 \mu \mathrm{g} / \mathrm{mL}) ; 6$-zoledronic acid $(20 \mu \mathrm{g} / \mathrm{mL})$.

standard solution.

\subsection{Linearity, Precision and Detection Limits}

Under the optimal experiment conditions, calibration curves for chloride, carbonate, sulfate, phosphate, hexametaphosphate, zoledronic acid were tested in a concentration range of 0.1 to $80.0 \mathrm{mg} / \mathrm{L}$. Each analyte showed good linearity with correlation coefficients no less than 0.9993 . Relative standard deviations (RSD, $n=10$ ) of the peak area and the correlation coefficients of the five analytes were shown in Table 2. The detection limit (LOD, signal-to-noise ratio of 3) of zoledronic acid was $0.030 \mu \mathrm{g} / \mathrm{mL}$. Compared with the reported LOD of zoledronic acid $(17 \mu \mathrm{g} / \mathrm{mL})$ [1], the sensitivity of the proposed method is much higher. From the values of RSD and the detection limits, it can be found that IC with potassium hydroxide self-regeneration suppressor and gradient eluent mode is a effective method for the analysis of zoledronic acid.

\subsection{Analysis of Zoledronic Acid in Injection Sample}

Real sample, zoledronic acid injection, was analyzed according to the above experimental conditions and the results were listed in Table 3 . Figure 2 shows the chromatogram of the real sample.

\section{Conclusion}

The proposed method represents improvements over prior reported techniques to quantitate zoledronic acid considered important in the management of the medicine. The calibration curves showed stability and matrix spiked recoveries were acceptable. Contents of zoledronic acid in the commercial injection sample were analyzed by means of IC using a gradient elution with potassium hydroxide self-regeneration suppressor and conductivity detection. The Chromatographic peaks of analytes were clearly separated. This method was applied to analyze 
Table 2. Performance of the proposed method.

\begin{tabular}{cccccc}
\hline Analytes & Regression equation $^{\mathrm{a}}$ & $\begin{array}{c}\text { Correlation } \\
\text { coefficient }\left(\mathrm{r}^{2}\right)\end{array}$ & $\begin{array}{c}\text { Linear range } \\
(\mu \mathrm{g} / \mathrm{mL})\end{array}$ & $\begin{array}{c}\mathrm{RSD}^{\mathrm{b}}(\mathrm{n}= \\
10, \%)\end{array}$ & $\begin{array}{c}\text { LOD }(\mathrm{S} / \mathrm{N}=3, \\
\mu \mathrm{g} / \mathrm{mL})\end{array}$ \\
\hline Chloride & $\mathrm{Y}=0.1381 \mathrm{X}-0.0025$ & 0.9994 & $0.1-80$ & 1.24 & 0.007 \\
Sulfate & $\mathrm{Y}=0.1284 \mathrm{X}+0.0012$ & 0.9999 & $0.1-80$ & 0.63 & 0.013 \\
Phosphate & $\mathrm{Y}=0.1037 \mathrm{X}-0.0005$ & 0.9998 & $0.1-50$ & 0.76 & 0.020 \\
Hexametaphosphate & $\mathrm{Y}=0.0911 \mathrm{X}+0.0036$ & 0.9993 & $0.1-50$ & 1.68 & 0.030 \\
Zoledronic acid & $\mathrm{Y}=0.0198 \mathrm{X}-0.0014$ & 0.9998 & $0.1-50$ & 1.07 & 0.030
\end{tabular}

${ }^{\mathrm{a} X} \mathrm{X}$ c concentration of analyte; Y: peak area; ${ }^{\mathrm{b}}$ The RSDs were calculated from replicate injections within one day.

Table 3. The results of analysis and recoveries of zoledronic acid in injection sample.

\begin{tabular}{cccccc}
\hline Analytes & $\begin{array}{c}\text { Found in the diluted } \\
\text { sample }(\mathrm{mg} / \mathrm{L})\end{array}$ & $\begin{array}{c}\text { Added } \\
(\mathrm{mg} / \mathrm{L})\end{array}$ & $\begin{array}{c}\text { Reclaimed } \\
(\mathrm{mg} / \mathrm{L})\end{array}$ & $\begin{array}{c}\text { Recovery } \\
(\%)\end{array}$ & $\begin{array}{c}\text { Concentration of analyte in original } \\
\text { injection sample }(\mathrm{mg} / \mathrm{L})\end{array}$ \\
Chloride & 546.15 & 500 & 1013 & 93.4 & 5461.5 \\
Sulfate & 1.26 & 2 & 3.32 & 103.0 & 12.6 \\
Phosphate & $\mathrm{ND}$ & 0.1 & 0.11 & 110.0 & $\mathrm{ND}^{\mathrm{a}}$ \\
Hexametaphosphate & $\mathrm{ND}$ & 0.1 & 0.09 & 90.0 & $\mathrm{ND}$ \\
Zoledronic acid & 4.01 & 10.00 & 14.13 & 101.2 & 40.1 \\
\hline
\end{tabular}

${ }^{\mathrm{a}}$ Not detectable.

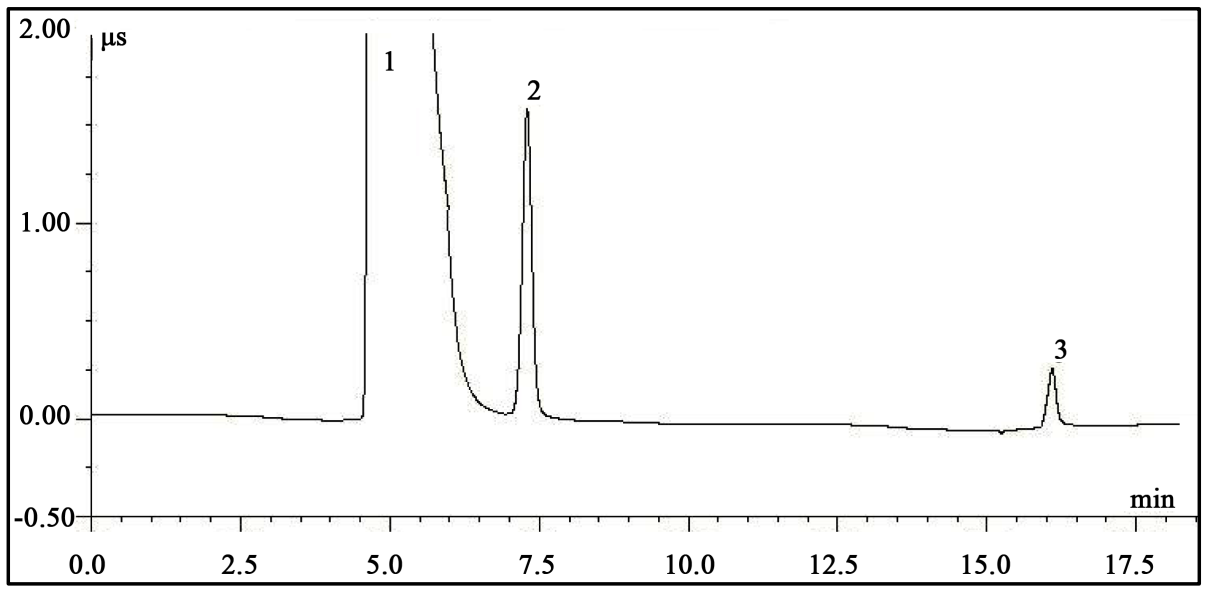

Figure 2. Chromatogram of the zoledronic acid injection sample; 1 -chloride; 2 -sulfate; 3 zoledronic acid.

real sample without interferences.

\section{Acknowledgements}

This research was financially supported by Zhejiang Provincial Natural Science Foundation of China (No. Z14B070002).

\section{References}

[1] Robert, E. (2004) Bisphosphonates: Clinical Experience. Oncologist, 9, 14.

[2] Major, P.P. and Cook, R. (2002) Efficacy of Bisphosphonates in the Management of Skeletal Complications of Bone Metastases and Selection of Clinical Endpoints. American Journal of Clinical Oncology, 25, 10. http://dx.doi.org/10.1097/00000421-200212001-00003

[3] Xie, Z., Jiang, Y. and Zhang, D.Q. (2006) Simple Analysis of four Bisphosphonates Simultaneously by Reverse Phase 
Liquid Chromatography Using N-Amylamine as Volatile Ion-Pairing Agent. Journal of Chromatography A, $1104,173$. http://dx.doi.org/10.1016/j.chroma.2005.11.113

[4] Rao, B.M., Srinivasu, M.K., Rani, C.P., kumar, S.S., Kumar, P.R., Chandrasekharc, K.B. and Veerender, M. (2005) A Validated Stability Indicating Ion-Pair RP-LC Method for Zoledronic Acid. Journal of Pharmaceutical and Biomedical Analysis, 39, 781. http://dx.doi.org/10.1016/j.jpba.2005.04.012

[5] Lovdahl, M.J. and Pietrzyk, D.J. (1999) Anion-Exchange Separation and Determination of Bisphosphonates and Related Analytes by Post-Column Indirect Fluorescence Detection. Journal of Chromatography A, 850, 143. http://dx.doi.org/10.1016/S0021-9673(99)00622-6

[6] Zhang, J.J. and Zhu, Y. (2007) Determination of Betaine, Choline and Trimethylamine in Feed Additive by Ion-Exchange Liquid Chromatography/Non-Suppressed Conductivity Detection. Journal of Chromatography A, 1170, 114. http://dx.doi.org/10.1016/j.chroma.2007.09.014

[7] Chen, M.L., Yan, W., Chen, Y.X., Jia, Z.S. and Zhu, Y. (2007) Determination of Impurities in Flame Retardant Monomer 2-Carboxyl Ethyl(Phenyl) Phosphinic Acid by Ion Chromatography. Journal of Chromatography A, $1155,47$. http://dx.doi.org/10.1016/j.chroma.2007.02.045 